\title{
SIZE REDUCTION OF DRUG PARTICLES BY PULSED LASER ABLATION TECHNIQUE
}

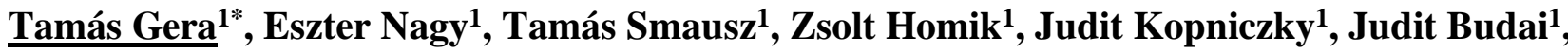 \\ Tibor Ajtai ${ }^{1}$, Rita Ambrus ${ }^{2}$, Piroska Szabó-Révész ${ }^{2}$, Béla Hopp ${ }^{1,3}$ \\ ${ }^{1}$ Department of Optics and Quantum Electronics, University of Szeged, \\ 6720 Szeged, Dóm square 9, Hungary \\ ${ }^{2}$ Institute of Pharmaceutical Technology and Regulatory Affairs, University of Szeged, \\ 6720 Szeged, Eötvös utca 6, Hungary \\ ${ }^{3}$ Department of Materials Science, Interdisciplinary Excellence Centre, University of Szeged, \\ 6720 Szeged, Dugonics tér 13, Hungary \\ *e-mail: gertamc2@gmail.com
}

DOI: https://doi.org/10.14232/kvantumelektronika.9.11

\section{Introduction}

Most of Non-Steroidal Anti-Inflammatory Drugs (NSAIDs) used in medication have poor water solubility and relatively low bioavailability. Many ongoing investigations aim to enhance the dissolution rate and solubility of these drugs and thereby reducing the required doses and diminishing the risk of side effects. To produce micro- or nanoparticles one of the most popular and efficient method is particle size reduction. [1] This method allows us to raise the specific surface of the particles which leads to advanced dissolution rate. For nanoparticle generation pulsed laser ablation (PLA) is an accepted and popular approach in case of both inorganic and organic materials. [2] Our former investigations have shown that PLAL/PLD is a proper technique for the size reduction of pharmaceutical particles without causing any chemical decomposition. [3-5] In this work we selected three NSAIDs as targets (ibuprofen, niflumic acid and meloxicam) with similar solubility, dissociation constant, particle size and crystallinity but with different chemical structures. We studied the effect of high-energy pulsed laser beam on the chemical degradation and the size distribution of the ablated drug particles. These model medicines are frequently used in acute pain therapy where fast absorption through the gastric mucosa is an essential requirement. The solubility of these pharmaceuticals is very poor in the gastric juice $(\mathrm{pH}=1.2)$ since they have a weak acidic character. However, ablated particles dissolve faster and can provide rapid analgesic effect. Our goal was to investigate the legitimacy of the PLA method using three different laser wavelengths $(248 \mathrm{~nm} ; 532$ $\mathrm{nm} ; 1064 \mathrm{~nm}$ ) for particle size reduction in ambient gas at standard pressure. We intended to produce submicron-sized drug particles, which may form the basis of a new type of pulmonary drug formulation for the rapid and efficient therapy of the hard-to-reach alveolar region of the lung. [6]

\section{Experimental}

We used commercially available pharmaceutical powders for producing target tablets by a hydraulic compactor at $175 \mathrm{MPa}$ pressure:

Ibuprofen ( $\alpha$-Methyl-4-(isobutyl) phenylacetic acid): white color, particle size of $15.3 \mu \mathrm{m}(\mathrm{d}(0.5))$, $75-77^{\circ} \mathrm{C}$ melting point, $157^{\circ} \mathrm{C}$ boiling point, $230{ }^{\circ} \mathrm{C}$ degradation temperature;

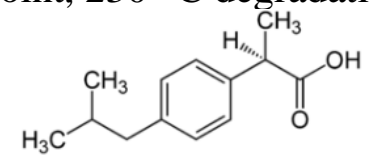

Meloxicam (4-hydroxy-2-methyl-N-(5-methyl-2-thiazolyl)-2H-benzothiazine-3-car-boxamide-1,1dioxide): yellow color, particle size of $3.78 \mu \mathrm{m}(\mathrm{d}(0.5)), 100 \%$ crystalline, $254{ }^{\circ} \mathrm{C}$ melting/decomposition temperature; 


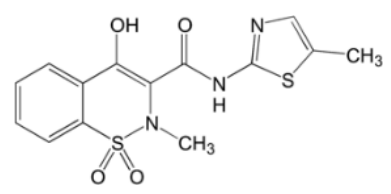

Niflumic acid (2-[ [3-(trifluoromethyl)phenyl] amino] -3-pyridinecarboxylic acid, white color, 18.85 $\mu \mathrm{m}(\mathrm{d}(0.5))$ particle size, $203{ }^{\circ} \mathrm{C}$ melting/decomposition temperature.<smiles>O=C(O)c1cccnc1Nc1cccc(C(F)(F)F)c1</smiles>

We investigated the optical absorption coefficient of these three pharmaceuticals by ellipsometry (Fig. 1). It can be seen that they have very diverse optical properties at the used wavelengths.

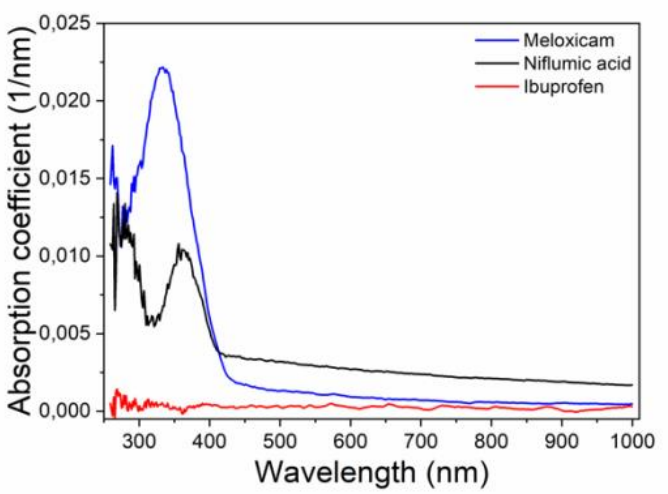

Figure 1. Absorption coefficients of ibuprofen (red), niflumic acid (black), and meloxicam (blue)

We irradiated the targets with high-energy $\left(1.5-15 \mathrm{Jcm}^{-2}\right)$ nanosecond laser impulses in $\mathrm{N} 2$ ambient gas at standard pressure using the following laser types: $\mathrm{KrF}$ excimer laser (FWHM=18 ns, $\lambda=248$ $\mathrm{nm}, 10 \mathrm{~Hz}$ repetition rate) and Nd:YAG laser (FWHM=6 ns, $\lambda=532 / 1064 \mathrm{~nm}, 10 \mathrm{~Hz}$ repetition rate). The laser beam was focused on the target surface at a grazing angle of $45^{\circ}$ by focusing lenses. We created a N2 gas flow along the chamber at constant flow rate $\left(2 \mathrm{lmin}^{-1}\right)$. The ablated particles were collected for FTIR analysis on a filter (pore size $1 \mu \mathrm{m}$, Merck Millipore Ltd. Omnipore Membrane Filter) (Fig. 2).

We have also investigated the size distribution of the generated particles by Scanning Mobility Particle Sizer (SMPS) by using the same experimental setup as it can be seen in Figure 2.

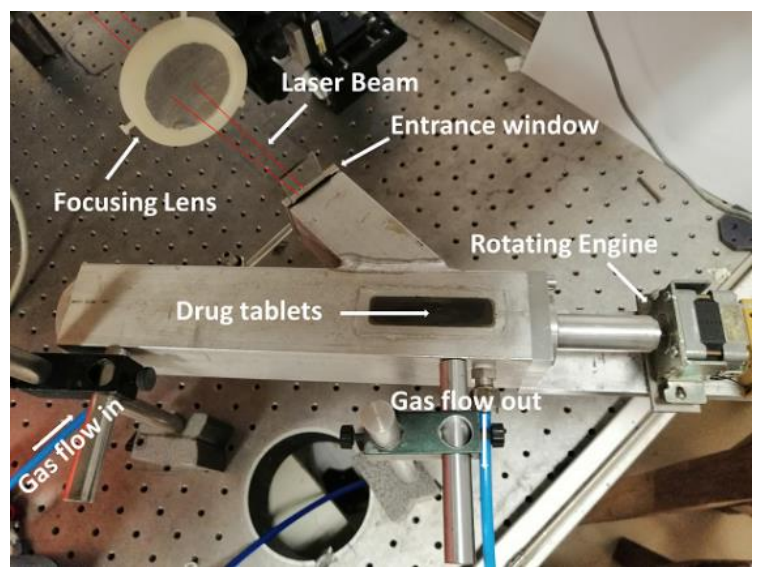

Figure 2. Experimental setup for PLA of drug pellets.

Moreover, we set up a generally applied pump and probe system (Fig. 3) for studying the material removal process. The pump laser was the above mentioned Nd:YAG laser and as a 
probe laser we implemented different nitrogen laser induced dye lasers adapting to the ablating wavelengths.

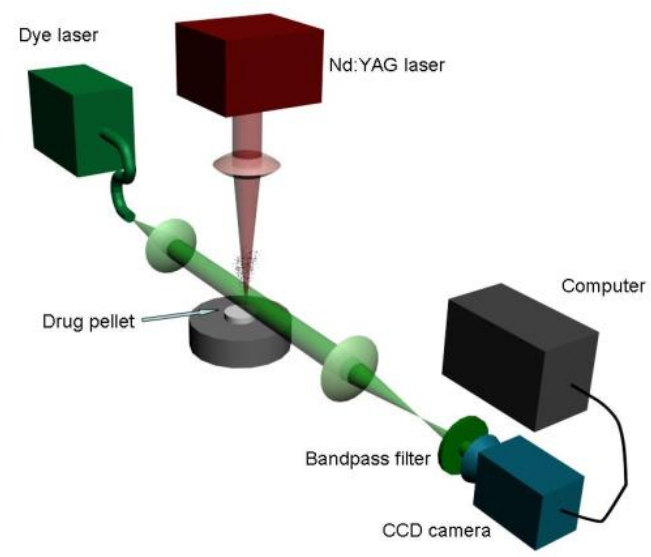

Figure 3. Time-resolved investigation of the particle ejections during PLA.

\section{Results and discussions}

\subsection{FTIR Spectroscopy for chemical characterization}

FTIR spectra of the ablated particles can be seen in Figure 4. It is observable that the ablation with UV (248 nm) laser beam resulted in destroyed particles while with visible (VIS) (532 nm) and IR $(1064 \mathrm{~nm})$ wavelengths chemically intact particles could be produced in the case of all three drugs. For further investigations we used only VIS and IR wavelengths since we intended to avoid chemical decomposition of the drugs.
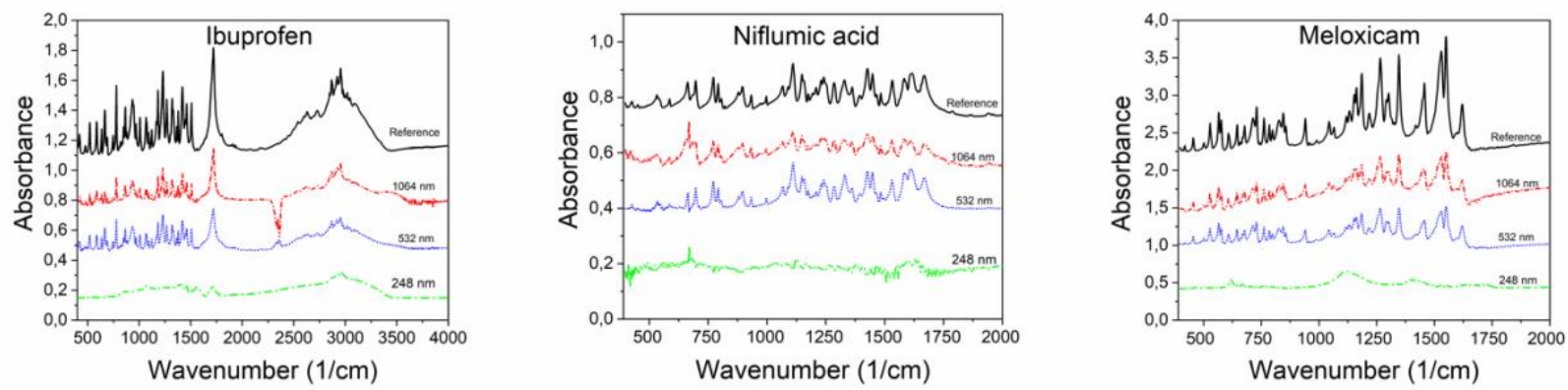

Figure 4. FTIR spectra of ablated drug particles in case of ibuprofen, niflumic acid and meloxicam

\subsection{Particle size characterization by SMPS measurements}

We investigated the particle size distributions of the ablated products produced at various experimental parameters. The ibuprofen particles were the smallest with having mode values smaller than $100 \mathrm{~nm}$ in all size distributions. It is followed by niflumic acid and then meloxicam with $\geq 100$ $\mathrm{nm}$ and $\sim 200 \mathrm{~nm}$ mode values, respectively. We concluded that no clear dependence on the laser fluence can be established.
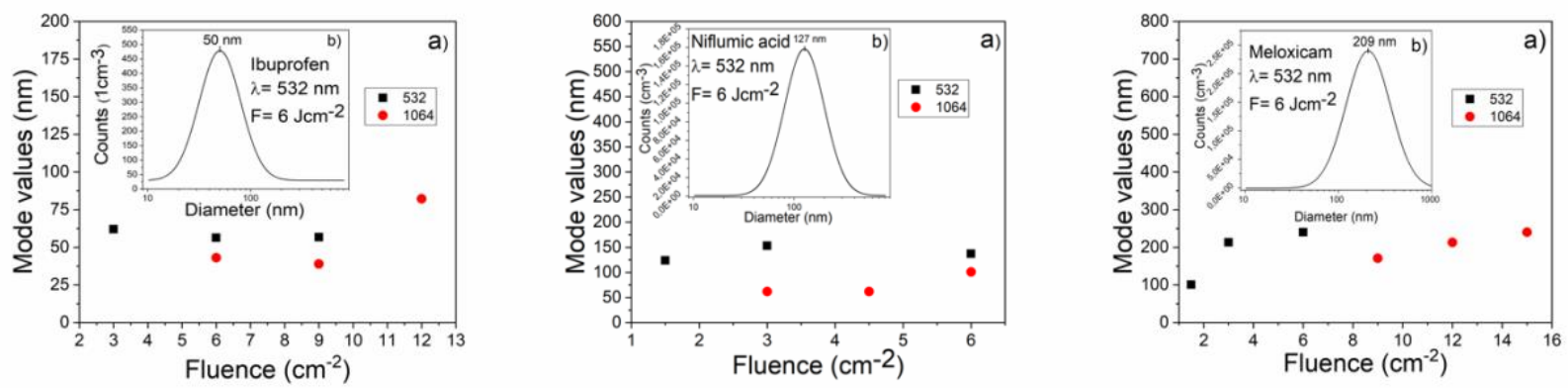
Figure 5. Particle-size measurements of ablated drug particles (ibuprofen, niflumic acid and meloxicam).

a) Mode values of the particle size distributions at various laser fluences; b) Particle size distributions at $532 \mathrm{~nm}$ laser wavelength and $6 \mathrm{Jcm}^{-2}$ fluence.

We calculated the penetration depth $\left(\mathrm{d}=\alpha^{-1}\right)$ of the laser light and the temperature rise in the target drug for VIS and IR laser impulses using the modified Beer-Lambert law (equation 1):

$$
\Delta T(x)=\frac{\alpha * F}{\rho * c} * e^{-\alpha x}(1),
$$

where $\alpha$ is the wavelength dependent absorption coefficient, $x$ is the distance from the surface, $F$ is the applied fluence of the laser beam, $\rho$ is the density and $c$ is the specific heat of the target material. Our calculations show that the temperatures inside the irradiated volume elements exceed well above $10^{3} \mathrm{~K}$ which means that the chemically intact particles cannot originate from these irradiated volumes. This indicates that these particles have to originate from the secondary effects of the ablation e.g. photomechanical effects.

\subsection{Time-resolved investigation of the particle ejections}

To understand and represent the dynamics of particle generation we captured fast photography images at different stages of the ablation process (Fig. 6) using VIS laser wavelength and $6 \mathrm{Jcm}^{-2}$ laser pulses. The ablation mechanism was similar for all three drugs. The laser beam induced micro-explosions at the target surface triggers the formation of shock waves. The produced shock wave is followed by an expanding cloud of matter in non-condensed state. Due to the recoil forces solid particles increasing in size with time start to exit the surface. We could not detect any particles larger than 1 $\mu \mathrm{m}$ with SMPS although it can be clearly seen that particles in all sizes were ablated. It can be related to the mass dependence of the mean free path and the limitation of the sizes of transportable particles by the magnitude of gas flow.

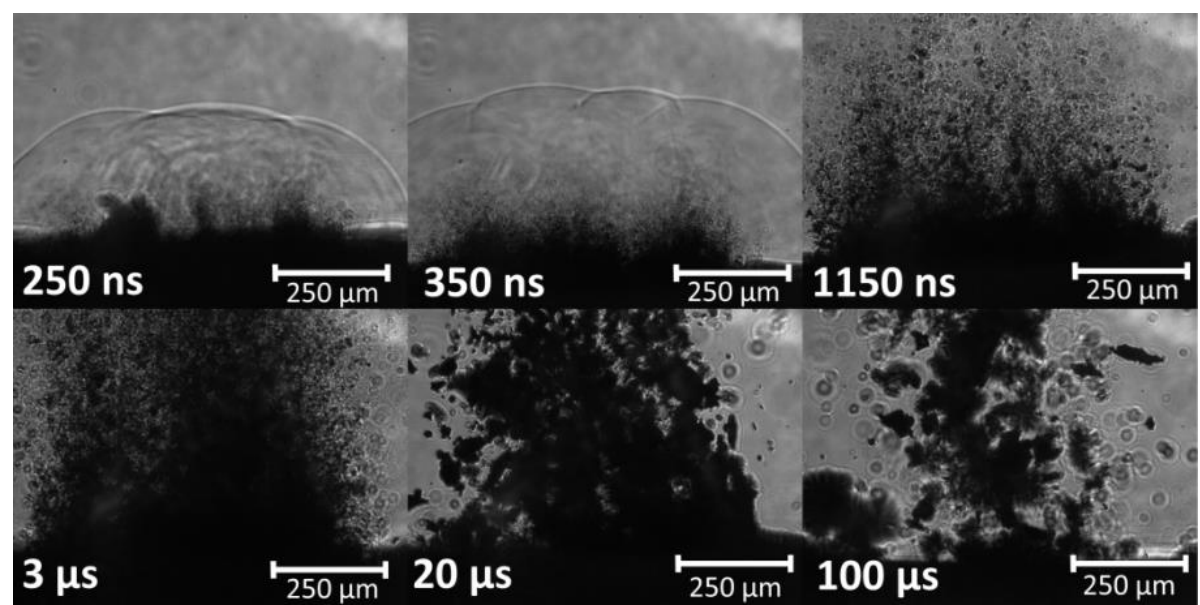

Figure 6. Time-resolved investigation of particle ejection in case of Niflumic acid using $532 \mathrm{~nm}$ laser wavelength and 6 $\mathrm{Jcm}^{-2}$ fluence.

We also estimated the magnitude of photomechanical effects by calculating (equation 2) the initial pressure produced at the shock front.

$$
P=\frac{2}{\gamma+1} * \rho * v^{2}(2)
$$

where $\mathrm{P}$ is the pressure produced at the shock front, $\mathrm{r}$ is the density of the medium, $\mathrm{v}$ is the velocity of the shock front and $\gamma$ is the proportion of specific heats $\left(\mathrm{c}_{\mathrm{p}} / \mathrm{cv}_{\mathrm{V}}\right)$ of the medium. The initial pressures at the shock fronts are closely equivalent with the recoil pressure of the shock wave what have 70$350 \mathrm{~atm}$ values. It verifies our assumption, that the photomechanical effects have a meaningful role in the ablation process of porous materials. 


\section{CONCLUSIONS}

We can state that PLA is a promising method for the size-reduction of ibuprofen, niflumic acid and meloxicam. By the selection of the laser parameters submicron to nanometer size particle managed to be generated, lowering the initial sizes of the drugs with orders of magnitude. Only VIS and IR laser wavelengths are suitable for producing chemically preserved molecules. The valuable nano- and submicron sized particles originate from the secondary photomechanical effects of the ablation.

We conclude that PLA can offer a chemical-free, reproducible and rather simple approach for the size reduction of the investigated NSAIDs. The produced submicron size particles can be beneficiate for the development of new pulmonary drug formulations for the medication of the lower lung area in pneumonia or severe acute respiratory syndrome. PLA can be advantageous over other generally applied practices by the adaptable laser parameters with the tuning of the grinding mechanism.

\section{ACKNOWLEDGEMENTS}

The project has been supported by the European Union, co-financed by the European Social Fund (EFOP-3.6.2-16-2017-00005); the Ministry of Human Capacities, Hungary grant (TUDFO/471381/2019-ITM FIKP program). T. Ajtai is indebted for his János Bolyai Research Scholarship of the Hungarian Academy of Sciences.

[1] Shegokar, R. \& Müller, R. H. Nanocrystals: Industrially feasible multifunctional formulation technology for poorly soluble actives. Int. J. Pharm. 399, 129-139 (2010).

https://doi.org/10.1016/j.ijpharm.2010.07.044

[2] Mele, A., Giardini-Guidoni, A. \& Teghil, R. Laser ablation of inorganic and organic materials. $J$. Chem. Sci. 105, 715-733 (1993).

https://doi.org/10.1007/BF03040845

[3] Hopp, B. et al. Production of meloxicam suspension using pulsed laser ablation in liquid (PLAL) technique. J. Phys. D. Appl. Phys. 51, 2018

https://doi.org/10.1088/1361-6463/aab4be

[4] Gera, T. et al. Production of ibuprofen in crystalline and amorphous forms by Pulsed Laser Deposition (PLD). Appl. Surf. Sci. 493, 359-367, 2019

https://doi.org/10.1016/j.apsusc.2019.06.254

[5] Ambrus, R. et al. Application of a suitable particle engineering technique by pulsed laser ablation in liquid (PLAL) to modify the physicochemical properties of poorly soluble drugs. J. Drug Deliv. Sci. Technol. 57, 101727, 2020

https://doi.org/10.1016/j.jddst.2020.101727

[6] Szabó-Révész, P. Modifying the physicochemical properties of NSAIDs for nasal and pulmonary administration. Drug Discov. Today Technol. 27, 87-93 2018

https://doi.org/10.1016/j.ddtec.2018.03.002 